\title{
Transmission Near-Infrared (NIR) and Photon Time-of-Flight (PTOF) Spectroscopy in a Comparative Analysis of Pharmaceuticals.
}

Kamran, Faisal; Abildgaard, Otto Højager Attermann; Sparén, Anders; Svensson, Olof; Johansson, Jonas; Andersson-Engels, Stefan; Andersen, Peter E.; Khoptyar, Dmitry

Published in:

Applied Spectroscopy

Link to article, DOI:

10.1366/14-07530

Publication date:

2015

Document Version

Publisher's PDF, also known as Version of record

Link back to DTU Orbit

Citation (APA):

Kamran, F., Abildgaard, O. H. A., Sparén, A., Svensson, O., Johansson, J., Andersson-Engels, S., Andersen, P. E., \& Khoptyar, D. (2015). Transmission Near-Infrared (NIR) and Photon Time-of-Flight (PTOF) Spectroscopy in a Comparative Analysis of Pharmaceuticals. Applied Spectroscopy, 69(3), 389-397. https://doi.org/10.1366/1407530

\section{General rights}

Copyright and moral rights for the publications made accessible in the public portal are retained by the authors and/or other copyright owners and it is a condition of accessing publications that users recognise and abide by the legal requirements associated with these rights.

- Users may download and print one copy of any publication from the public portal for the purpose of private study or research.

- You may not further distribute the material or use it for any profit-making activity or commercial gain

- You may freely distribute the URL identifying the publication in the public portal 


\title{
Transmission Near-Infrared (NIR) and Photon Time-of-Flight (PTOF) Spectroscopy in a Comparative Analysis of Pharmaceuticals
}

\author{
Faisal Kamran,, ${ }^{a, \star}$ Otto H.A. Abildgaard,b Anders Sparén, ${ }^{c}$ Olof Svensson, ${ }^{c}$ \\ Jonas Johansson, ${ }^{c}$ Stefan Andersson-Engels, ${ }^{d}$ Peter E. Andersen, ${ }^{a, \star}$ Dmitry Khoptyard \\ a Department of Photonics Engineering, Technical University of Denmark, Frederiksborg 399, 4000 Denmark \\ b Department of Informatics and Mathematical Modelling, DTU, Asmussens All 321, 2800 Denmark \\ c Department of Pharmaceutical Development, AstraZeneca R\&D, Mölndal, SE-431 83, Sweden \\ d Department of Physics, Lund University, P. O. Box 118, Lund SE-221 00, Sweden
}

\begin{abstract}
We present a comprehensive study of the application of photon time-of-flight spectroscopy (PTOFS) in the wavelength range 1050$1350 \mathrm{~nm}$ as a spectroscopic technique for the evaluation of the chemical composition and structural properties of pharmaceutical tablets. PTOFS is compared to transmission near-infrared spectroscopy (NIRS). In contrast to transmission NIRS, PTOFS is capable of directly and independently determining the absorption and reduced scattering coefficients of the medium. Chemometric models were built on the evaluated absorption spectra for predicting tablet drug concentration. Results are compared to corresponding predictions built on transmission NIRS measurements. The predictive ability of PTOFS and transmission NIRS is comparable when models are based on uniformly distributed tablet sets. For non-uniform distribution of tablets based on particle sizes, the prediction ability of PTOFS is better than that of transmission NIRS. Analysis of reduced scattering spectra shows that PTOFS is able to characterize tablet microstructure and manufacturing process parameters. In contrast to the chemometric pseudovariables provided by transmission NIRS, PTOFS provides physically meaningful quantities such as scattering strength and slope of particle size. The ability of PTOFS to quantify the reduced scattering spectra, together with its robustness in predicting drug content, makes it suitable for such evaluations in the pharmaceutical industry.
\end{abstract}

Index Headings: Spectroscopy; Scattering measurements; Turbid media; Multiple scattering; Spectroscopy, condensed matter.

\section{INTRODUCTION}

The manufacturing process of pharmaceutical materials involves high-quality requirements to ensure efficiency and patient safety. Process analytical technology has been defined by the United States Food and Drug Administration as a mechanism to design, analyze, and control pharmaceutical manufacturing processes. ${ }^{1}$ Nearinfrared spectroscopy (NIRS) is a powerful spectroscopic technique widely employed in the pharmaceutical industry to monitor different stages of the production process and to analyze the final products. ${ }^{2-5}$ Since its successful online implementation by major pharmaceutical companies, NIRS has been widely used in critical

Received 18 March 2014; accepted 12 September 2014.

* Authors to whom correspondence should be sent. E-mail: faisal309@ gmail.com; peta@fotonik.dtu.dk.

DOI: $10.1366 / 14-07530$ stages of the pharmaceutical process for the characterization of raw materials, drying, blending, and tableting. ${ }^{6}$

Near-infrared spectroscopy allows relatively cheap, rapid, easy, and non-destructive measurements, without the need for sample preparation. ${ }^{7}$ Its analytical power has been demonstrated in a number of qualitative and quantitative pharmaceutical applications. ${ }^{8}$ Chemometric methods have been applied to NIR spectroscopic data by the pharmaceutical industry to determine drug contents, ${ }^{9}$ polymorphic content, ${ }^{10}$ and particle sizes of powders. ${ }^{11,12}$

The key challenge in NIRS is to develop methods that not only are accurate enough to determine analytical parameters, such as content uniformity between different tablets, but also have an inherent robustness such that the prediction of concentration is insensitive to variations in, e.g., raw material particle size and batch quality. ${ }^{13}$ The inherent high correlation of spectral shapes and thus difficulty and inability to distinguish the contributions from such parameters in NIRS spec$\operatorname{tra}^{14,15}$ require the development and maintenance of extensive calibration databases. NIRS transmission spectra depend on both the structural and chemical properties of the medium. Elastic scattering of nearinfrared light in a pharmaceutical tablet dominates over absorption by several orders of magnitude. Thus, the NIR spectra are strongly susceptible to parameters related to light scattering. This makes independent determination of particle size of major importance. ${ }^{16}$ Abrahamsson et al. have shown that predictions based on NIRS data were improved by correcting for scattering effects in the measured spectra. ${ }^{17} \mathrm{~A}$ method that is insensitive to variations in the scattering properties of the medium is highly desirable. It is widely recognized ${ }^{18}$ that reduced scattering coefficient can be significantly changed by variation in microstructure of turbid media. To correct for the influence of structural properties, NIR transmission spectra normally have to be preprocessed before they can be analyzed. ${ }^{19,20}$

Photon time-of-flight spectroscopy (PTOFS) ${ }^{17}$ is a spectroscopic technique in which the temporal distribution of photon propagation times through samples is measured. It provides less interdependent information on reduced scattering (influenced by the structural properties) and absorption (influenced by the chemical composition) of a turbid medium. PTOFS is becoming increasingly popular in broadband instruments. ${ }^{21-23}$ The technique has been used specifically for the inspection 


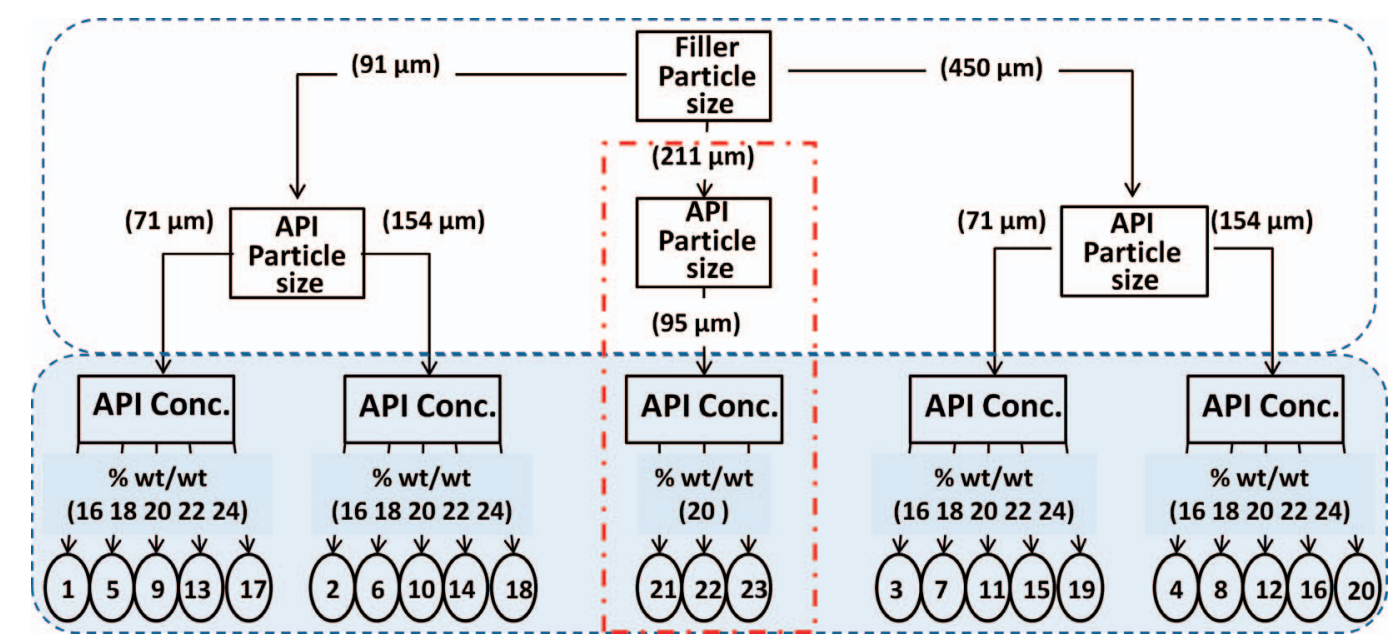

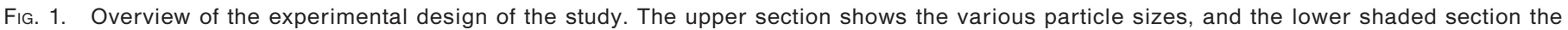

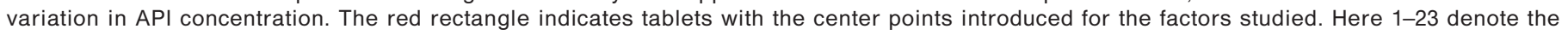
combinations of factors.

of tablets ${ }^{24-26}$ and powders. ${ }^{27}$ Khoptyar et al. ${ }^{26}$ have recently shown feasibility of spectroscopic evaluation of tablet concentration on the basis of their PTOFS absorption spectra. Because of the small number of investigated tablets, it was impossible to consider robustness of the technique and its sensitivity to large scattering variation due in part to varying scatterer size and different tablet compaction force. So far, little has been done to systemically study the potential and limitations of the new generation of broadband PTOFS system for tablet spectroscopic analysis.

In this paper, we demonstrate that PTOFS provides a valuable addition to spectroscopic analysis of pharmaceuticals in the wavelength range 1050-1350 nm. Broadband PTOFS was applied in a comprehensive study of 75 tablets in which the drug concentration, filler, and API particle size and compression force were varied. The ability of PTOFS to measure drug concentration was investigated in terms of the prediction ability. The results obtained were compared to those obtained with continuous-wave, transmission NIRS. The robustness of PTOFS toward particle size variation is investigated and compared to transmission NIRS. Finally, it is shown that PTOFS reduced scattering spectra can be used to determine particle form, size, and compression force, without a chemometric calibration model.

\section{MATERIALS AND METHODS}

Materials. The 75 tablets used in this study were composed from three components: mannitol, ibuprofen, and magnesium stearate. The active pharmaceutical ingredient (API) in the tablets was ibuprofen, and mannitol was used as the filler. Magnesium stearate is a lubricant added to facilitate tablet compression. The tablets were made by mixing the ingredients according to the experimental design shown in Fig. 1. The experiment was designed to investigate individual and interdependent variation by varying the following factors: filler particle size (FPS), API particle size, API concentration, and filler particle manufacturing method. The complete set of combinations consists of 23 unique samples, denoted 1 to 23 , spanning a wide range of material compositions. The FPSs (mass median diameter) used in the design were 91,211 , and $450 \mu \mathrm{m}$, respectively. ${ }^{28}$ The average particle diameters of ibuprofen used in the design were 71, 95, and $154 \mu \mathrm{m}$, respectively, and the average concentration was varied at five levels; $16,18,20,22$, and $24(\% \mathrm{w} / \mathrm{w})$, respectively. Properties for different filler sizes and the manufacturing methods are presented in Table I.

All the tablets were pressed with a single punch press (Korsch EK 0, Korsch AG, Berlin, Germany) equipped with flat, round $10 \mathrm{~mm}$ punches. The compression force (CF) was set manually to 8,12 , and $16 \mathrm{kN}$ denoted CF1, CF2, and CF3, respectively, for all powder blends resulting in $3 \times 23$ different tablet types (batches). Single-component tablets were compressed in a manual tablet press (Specac, Oprington, UK) using a CF of approximately $20 \mathrm{kN}$. All tablets had a uniform diameter of $10 \mathrm{~mm}$, with a thickness of $\sim 3 \mathrm{~mm}$ and weight of $\sim 300 \mathrm{mg}$. The tablets were manufactured at AstraZeneca R\&D Mölndal, Sweden, for another study. ${ }^{29}$

Transmission Near-Infrared Spectroscopy. Transmission NIRS spectra were recorded for all the tablets using a Fourier transform NIR spectrometer (MB160, ABB-Bomem Inc., Canada), in transmission mode. ${ }^{29}$ The spectra were recorded as the average of 32 scans for each tablet, and the resolution was set to $16 \mathrm{~cm}^{-1}$ corresponding to $\sim 2 \mathrm{~nm}$ at $1200 \mathrm{~nm}$. For a more direct comparison between transmission NIRS and PTOFS the wavelength region from 1050 to $1350 \mathrm{~nm}$ was used. For comparative study, the API concentrations in the samples were determined using Raman spectroscopy. ${ }^{29}$

TABLE I. Properties of the different filler particle sizes (FPSs). ${ }^{28}$

\begin{tabular}{llcc}
\hline Particle size $(\mu \mathrm{m})$ & Method & BD $(\mathrm{g} / \mathrm{ml})$ & Comp. $(\%)$ \\
\hline FPS1 (91) & Spray dried & 0.57 & 6.9 \\
FPS2 (450) & Granular & 0.71 & 4.5 \\
FPS3 (211) & Spray dried & 0.50 & 9.9 \\
\hline
\end{tabular}

$\mathrm{BD}=$ bulk density; Comp. = compressibility. 


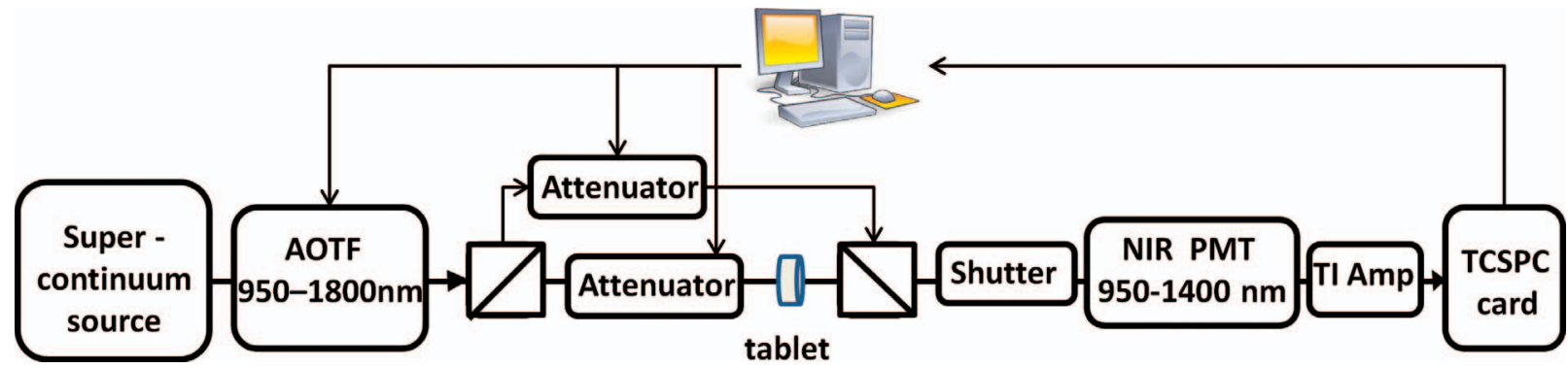

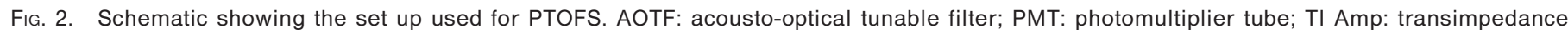
amplifier; TCSPC card: time-correlated single-photon counting card.

Photon Time-of-Flight Near-Infrared Spectroscopy. In PTOFS, the time-distribution of photons transmitted through a sample is evaluated with picosecond time resolution. A schematic of the PTOFS system is shown in Fig. 2. A photonic crystal fiber supercontinuum source (SuperK Extreme EXW 12, NKT Photonics A/S, Birkerød, Denmark) was used in combination with an AOTF (SuperK Select, NKT 384 Photonics A/S, Birkerød, Denmark) to generate tunable probe pulses with which to irradiate the tablet. A small fraction of the pulse power is split off prior to the sample and routed directly to the detector for timing reference to increase measurement accuracy. ${ }^{26}$ A photomultiplier tube (Hamamatsu, model R3809U-68) is used for detection. The signal is fed to a time-correlated single-photon counting (TCSPC) electronics (Becker\&Hickl, model SPC-130) for high-precision measurements of the PTOF distribution. ${ }^{26}$ Data acquisition is carried out using an automated measurement sequence implemented in $\mathrm{CH}$. The measurement routine involves setting up the TCSPC module and setting the wavelength and amplitude of the light source. The attenuation of the light intensity through samples was adjusted to obtain a count rate up to $50 \mathrm{kHz}$ during data acquisition. A total photon count of more than 50000 was considered sufficient for each data point, yielding a recording time of $\sim 30 \mathrm{~s}$ per measurement. Tablet spectra were scanned in the spectral range 950$1400 \mathrm{~nm}$ with $4 \mathrm{~nm}$ resolution. The sequence in which tablets were investigated was also randomized in order to avoid any bias.

The shape of the PTOF distribution is affected by the absorption and scattering of light, which are directly influenced by the chemical and structural composition of the medium. The optical properties, i.e., absorption and reduced scattering, were evaluated from the PTOF distribution at each wavelength with Matlab, using a Levenberg-Marquardt-based fitting algorithm with the diffusion approximation model developed by Contini and Martelli et al. ${ }^{30}$ Black-printed office paper is used as a sample to get instrument response function. ${ }^{21}$ The instrument response function was in all subsequent evaluations convolved with the fitted diffusion model to take into account the light dispersion in the optical fibers and finite detector response time. The estimated absorption coefficient, $\mu_{a}$, is normally used for the analysis of the chemical constituents and the composition of the tablet. The reduced scattering coefficient, $\mu_{s}^{\prime}=\mu_{s}(1-g)$, is generally correlated to the structural properties, where $\mu_{s}$ is scattering and $g$ is called the anisotropy of the medium.

The signal-to-noise ratio (SNR) for both transmission NIRS and PTOFS was calculated by fitting a polynomial to a section of each spectrum, using the expression:

$$
\mathrm{SNR}=\operatorname{mean}\left[\frac{\text { spectra }}{\mid \text { Polynomial fit }- \text { spectra } \mid}\right]
$$

\section{DATA ANALYSIS}

Multivariate regression analysis ${ }^{31}$ was performed using partial least squares regression ${ }^{32}$ to predict the API concentrations from transmission NIRS and PTOF spectra, respectively. Calibration models for the API content were constructed by dividing the data into calibration and validation sets. Group-fold cross-validation $^{32}$ and manual selection were used to determine the number of latent variables in the calibrations models. The calibrations were then applied to the validation set to predict the API concentration. In the first case, $70 \%$ of the samples were randomly chosen as the calibration set, and the remaining $30 \%$ served as the validation set. In the second case, the data were divided based on the average particle size of the tablets.

Analysis of Transmission Near-Infrared Spectra. Near-infrared spectra often require pretreatment for underlying scattering signal. ${ }^{19}$ The effect of difference in particle sizes on TNIRS spectra is quantitatively the most important. It can partly be corrected using mean centered data, ${ }^{31}$ derivatives, ${ }^{33}$ multiplicative scatter correction (MSC), ${ }^{34}$ or standard normal variate. ${ }^{35}$ We used MSC for the preprocessing of the transmission NIRS spectra.

Analysis of Photon Time-of-Flight Data. The PTOFS technique provided spectra of absorption and reduced scattering as described above. Principal component analysis (PCA) was used in evaluating the absorption spectra, as this provides the most compact orthogonal description of the data. ${ }^{31}$ Reduced scattering spectra were modeled with an approximation from Lorentz-Mie theory as explained later.

\section{RESULTS AND DISCUSSION}

Measurement Stability and Noise. Bias-variance tests were carried out to determine the stability of the PTOFS system. Figure $3 a$ shows the absorption coefficients of a tablet estimated at three wavelengths close to 


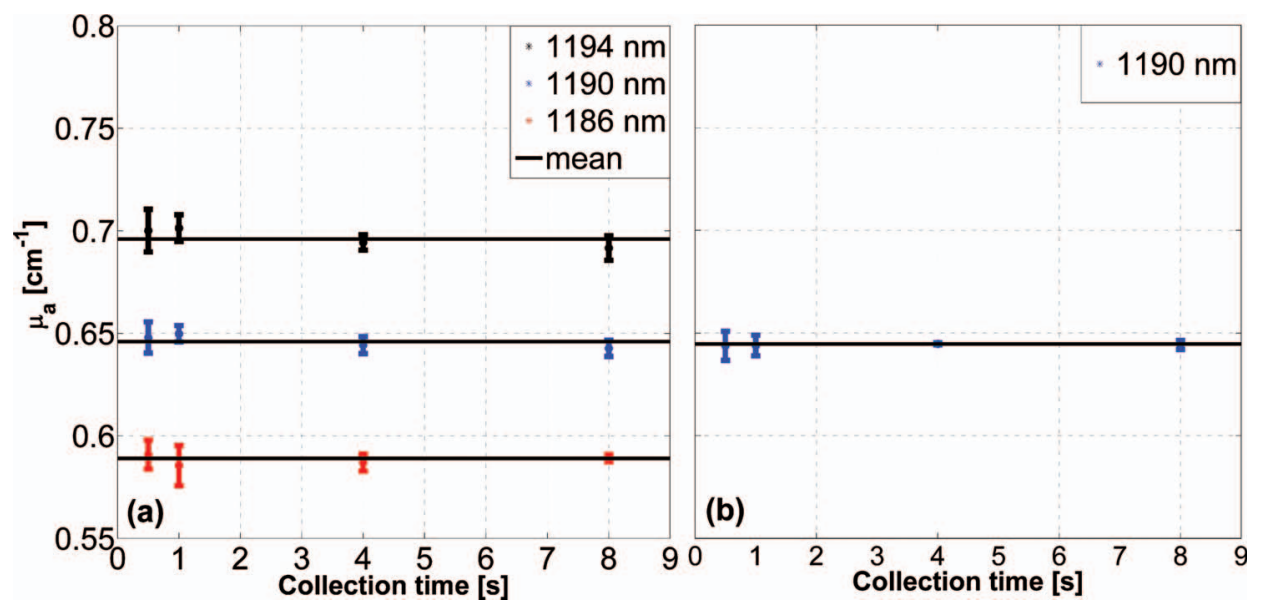

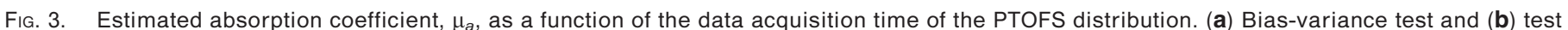

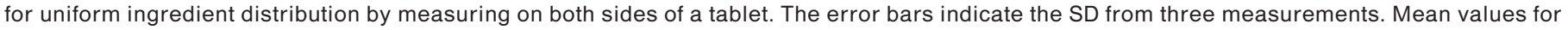
each wavelength are also plotted.

the absorption peak, as a function of data acquisition time. Error bars are the result of repetitive measurements. The acquisition time for the PTOF distribution was varied in these measurements between 0.5 and $8 \mathrm{~s}$ at each wavelength. The evaluated absorption for each measurement is within the standard deviation (SD) from the mean of all measurements at that wavelength. The absorption coefficients obtained are constant within $1 \%$, showing that no bias was introduced by altering the acquisition time. Similar tests were performed to check the variation in the distribution of ingredients inside the tablets. A tablet was measured twice from both sides, and the system showed reliable and reproducible results, as can be seen in Fig. 3b.

Evaluated Absorption and Active Pharmaceutical Ingredient Concentrations. Absorbance and absorption spectra from both the transmission NIRS and PTOFS technique are presented in Fig. $4 \mathrm{a}$ and $4 \mathrm{~b}$, respectively. The transmission NIRS attenuation spectra are plotted in the same wavelength range as the PTOFS spectra, and it can be seen that there is, in general, good agreement between the two methods. The influence of the scattering properties of the samples can be visualized by a prominent offset shift in the transmission NIRS spectra. The SNR, calculated from Eq. 1, was more than 10 times higher in the transmission NIRS spectra than in the PTOFS spectra. Spectra of pure ingredients are also shown in Fig. 4b, which indicates an overlap of mannitol and ibuprofen spectra at $1200 \mathrm{~nm}$.

Figure 5 shows the scatter plot resulting from the PCA applied to PTOFS absorption spectra for the first two principal components. Ideally separated absorption spectra from reduced scattering spectra will not show any influence from the particle size. If labeled for an uncompressed FPS, no data grouping will be observed. However, the scatter plot shows that tablets with larger filler particles cluster separately from the others, indicating that absorption is not completely separated from reduced scattering. This correlation can be explained by the crosstalk between the optical properties ${ }^{36}$ and requires further investigation. For the PTOFS data the first two principal components explain more than $80 \%$ of the variance. The results of PCA also suggested that the wavelength region between 1050 and $1350 \mathrm{~nm}$

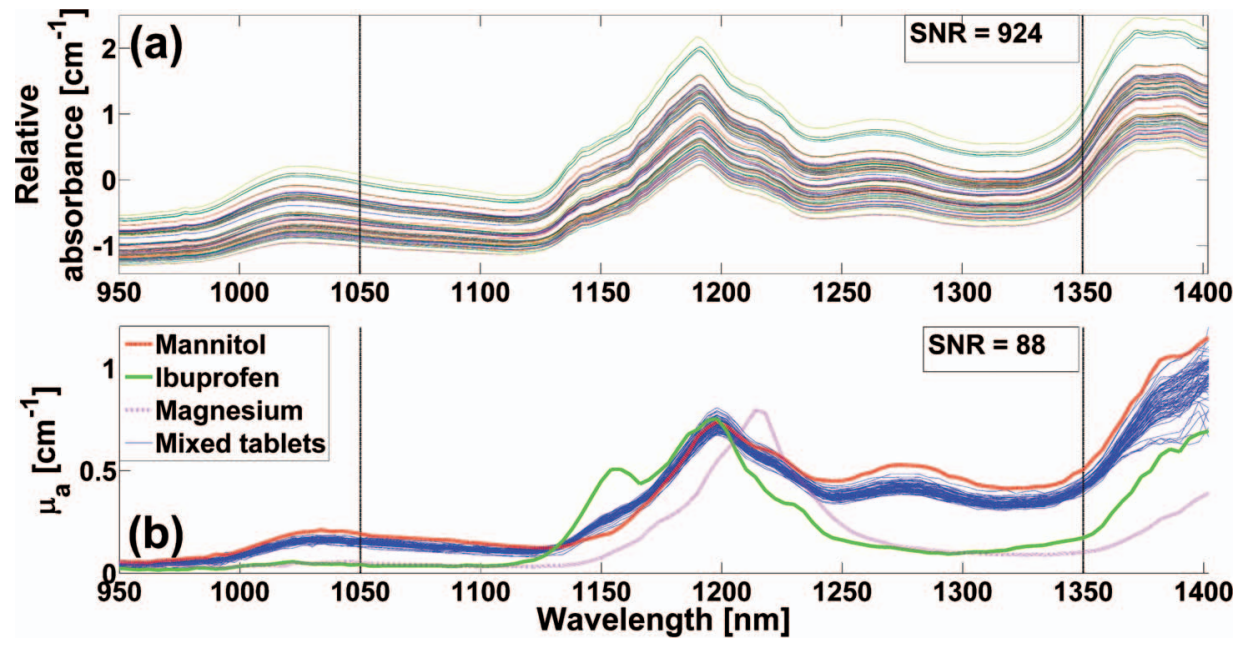

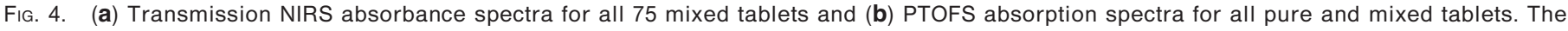
dashed vertical lines indicate $1050-1350 \mathrm{~nm}$ used for analysis. The signal-to-noise ratio (SNR) is given in each case. 


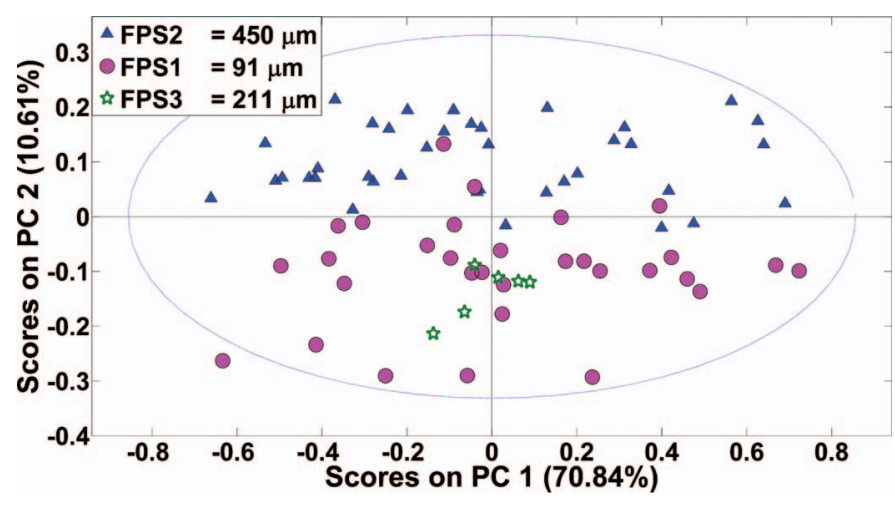

FIG. 5. PCA scatter plot for tablets with different FPSs (before compression) on the first two principal components (PC) for PTOFS absorption spectra. The dashed ellipse indicates $95 \%$ confidence interval.

was suitable for prediction of API content since the variation in concentration could be clearly seen in the first principal component.

To predict the API concentrations from both transmission NIRS and PTOFS spectra, partial least squares regression was used, relating the variance to the desired output. The prediction is achieved by extracting a set of orthogonal factors called latent variables from the predictors (wavelengths) that have the best predictive power. The contribution toward variance by predictors for each latent variable is called loading, which can explain how variance may relate to the predictions. Loadings of the first six latent variables of partial least squares for transmission NIRS and PTOFS spectra are shown in Fig. $6 a$ and 6b, respectively. Out of those six, physically meaningful information is contained in only two for transmission NIRS and four for PTOFS. In the case of transmission NIRS, two latent variables are necessary to describe ibuprofen concentrations. For PTOFS, not only ibuprofen concentration and mannitol amplitude is explained, but the crosstalk by scattering as well is captured, requiring comparatively complex models (four variables). Therefore, two and four latent variables are used for transmission NIRS and PTOFS predictions, respectively.

Predictive models are built on tablets in a calibration set and then validated on tablets in a validation set.
Tablet data are first split into $70-30 \%$ sets for calibration and validation, respectively. The prediction performance was then compared to well-structured classification of data. Table II presents the average results of a 100 random set of calibration and validation samples. Ratio of performance to deviation (RPD) is a measure of the usefulness of models, and a value more than three is considered useful for screening. ${ }^{37}$ Four latent variables for the PTOFS data produced RPD $=3$ and root mean square error of prediction, ${ }^{38}$ RMSEP $=0.92$. These should be compared with RPD $=2.77$ and RMSEP $=1.03$ when using two latent variables for the transmission NIRS data. Variance explained by latent variables $\left(R^{2}\right)$ in both cases is comparable. The reasons for PTOFS higher model complexity are the inherent sparseness of the experimental design of the tablet data set and the crosstalk between optical properties.

To compare the robustness of the two techniques the models are challenged by selecting a validation set based on different FPSs than those of the calibration set. The purpose of challenging the models in this way is to see if PTOFS is less sensitive toward variations in raw material than transmission NIRS. Table III presents the prediction results. The models were calibrated using data sets with large FPS, and the predictions were validated on data sets with a small FPS. Model complexities are kept similar to the previous case. Compared to results in Table II, models based on PTOFS spectra improved the RPD value to 3.42 and reduced the RMSEP to 0.84. Models on transmission NIRS data, however, showed higher sensitivity to variations in particle sizes, thus making predictions more difficult. The difference in RMSEP values for transmission NIRS (1.12) and PTOFS (0.84) is statistically significant.

The prediction accuracy of both techniques is comparable when the models are based on uniformly distributed particle sizes. However, when the models are based on the data set using one particle size and the predictions are made for different particle sizes, the prediction error for PTOFS is smaller than that with transmission NIRS. PTOFS thus showed less sensitivity to the variations in raw filler material than transmission NIRS. It can therefore be concluded that the analysis using PTOFS is more robust toward changes in particle sizes.
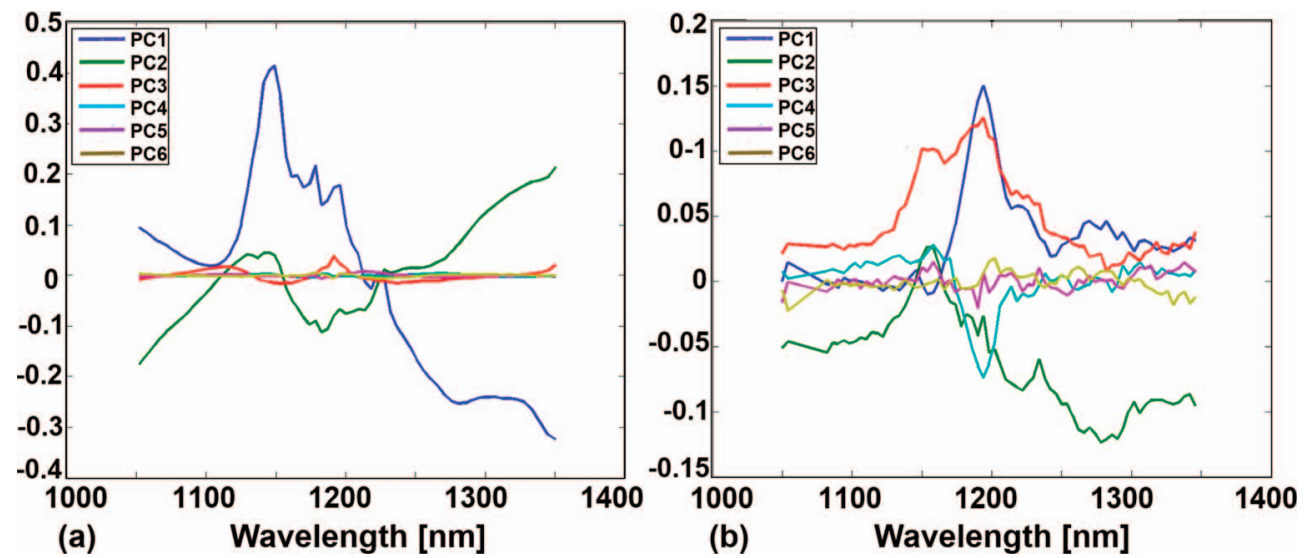

FIG. 6. Loadings of the first six latent variables of partial least squares for (a) transmission NIRS data and (b) PTOFS data. 
TABLE II. Results of partial least squares regression using group-fold cross-validation for API concentration prediction from transmission NIRS and PTOFS data. The average values over 100 runs are given for the random $70-30 \%$ split of the data. The same spectral region, $1050-$ $1350 \mathrm{~nm}$, was considered for both techniques. ${ }^{a}$

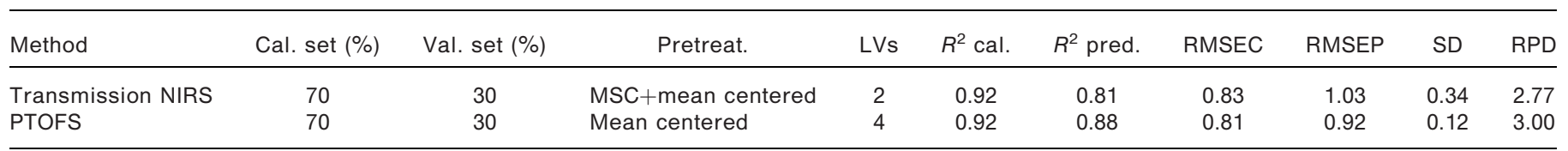

${ }^{a}$ Cal. = calibration; Val. = validation; Pretreat. = pretreatment; LVs = latent variables; $\mathrm{R} 2=$ Variance explained; RMSEC $=$ root mean square error of calibration; RMSEP = root mean square error of prediction; SD = standard deviation; RPD = ratio of performance to deviation.

TABLE III. Results of partial least squares regression using group-fold cross-validation for API concentration prediction from transmission NIRS and PTOFS data. Model generalization tested on structured selection of calibration and validation sets based on average particle sizes.

\begin{tabular}{|c|c|c|c|c|c|c|c|c|c|c|}
\hline Transmission NIRS & Large particles & Small particles & $\mathrm{MSC}+$ mean centered & 2 & 0.92 & 0.86 & 0.85 & 1.12 & - & 2.57 \\
\hline PTOFS & Large particles & Small particles & Mean centered & 4 & 0.93 & 0.90 & 0.77 & 0.84 & - & 3.42 \\
\hline
\end{tabular}

Photon Time-of-Flight Spectroscopy Reduced Scattering. Figure 7 presents PTOFS reduced scattering spectra for pure ingredients and mixed tablets, respectively. Although the absorption and reduced scattering have different origins, they are often correlated ${ }^{36}$ due to crosstalk as a result of finite bandwidth, modeling inaccuracies, or data-gathering procedures. The reduced scattering spectra from mixed tablets indicate a correlation with absorption peaks around $1200 \mathrm{~nm}$ and after $1350 \mathrm{~nm}$ (see Fig. 4). All the mixed tablets exhibit higher wavelength dependence of the scattering slope and scattering amplitudes than ibuprofen and magnesium stearate. This indicates that mannitol is mainly responsible for scattering inside the mixed tablets. The reduced scattering coefficient is defined as ${ }^{39}$

$$
\mu_{s}^{\prime}=\rho Q_{s} \frac{\pi d^{2}}{4}(1-g)
$$

where $\rho$ is the average particle number density, $Q_{s}$ is size-dependent scattering efficiency of the particle, $d$ is diameter of the scatterer, and $g$ is the anisotropy of the medium. The scattering efficiency $Q_{s}$ tends to approach a value of 2 for large pure scatterers, for $\pi d / \lambda \gg 1$, where $\lambda$ is the wavelength of the light. The empirically observed scattering behavior for small and intermediate sized particles is relatively well modeled using Rayleigh and Mie theories: ${ }^{40}$

$$
\mu_{s}^{\prime}=A\left(\lambda / \lambda_{0}\right)^{-\beta}
$$

Equation 3 is a relatively good approximation for scattering behavior of pharmaceuticals in the NIR spectral regime. $A$ is the reduced scattering coefficient at the reference wavelength $\lambda_{0}$, and $\beta$ is the slope of the fit versus wavelength. For Rayleigh scattering, the size parameter, $\pi d / \lambda$, is much less than 1 , and the scattering is dependent on $\lambda^{-4}$. For larger particles than the Rayleigh limit, $\beta$ varies from 4 to 0 depending on the size parameter. The large particle limit is $\beta=0$, where $Q_{s}=2$. At such size parameters $\mu_{s}^{\prime}$ will be independent of wavelength. According to Lorentz-Mie scattering calculations, the filler particles would approach this limit for all three FPSs. However, the reduced scattering of mannitol is highly dependent on wavelength, indicating an effective size parameter being much smaller than for the original mannitol particles used for the production of the tablets. Figure 8 shows scanning electron microscopy images of original filler particles with three different FPS used in the tablets, where it can be seen that they

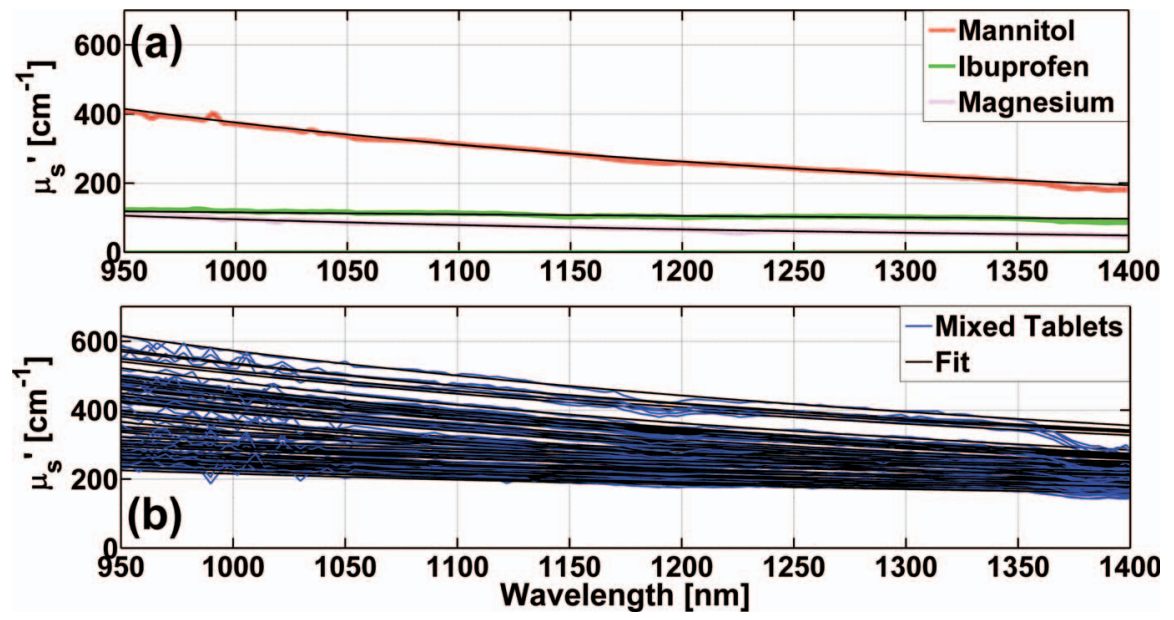

FIG. 7. (a) Reduced scattering spectra $\left(\mu_{s}^{\prime}\right)$ for pure ingredients and (b) mixed tablets. The black curves are the predictions based on Eq. 3. 


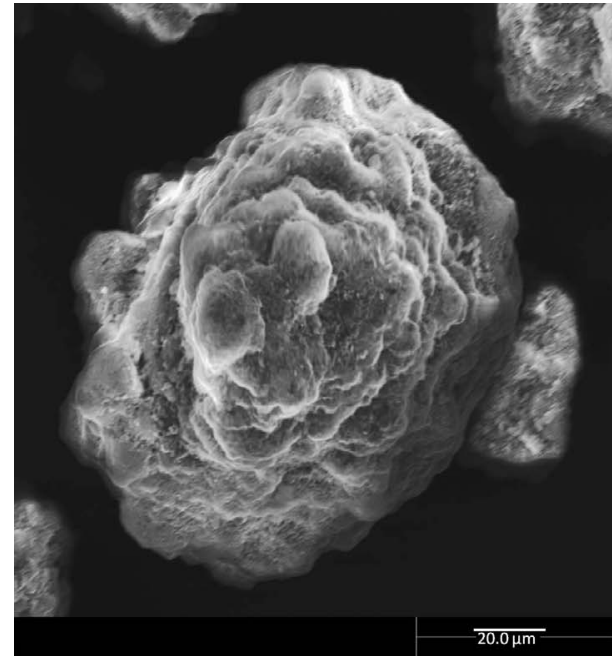

(a)

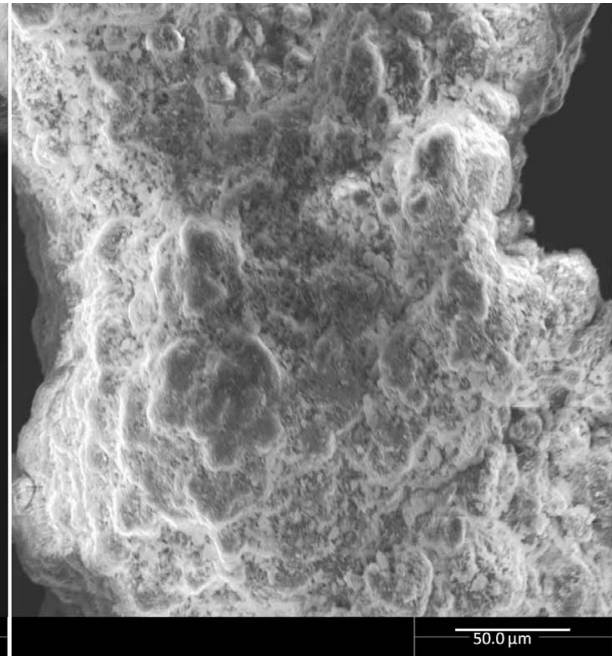

(b)

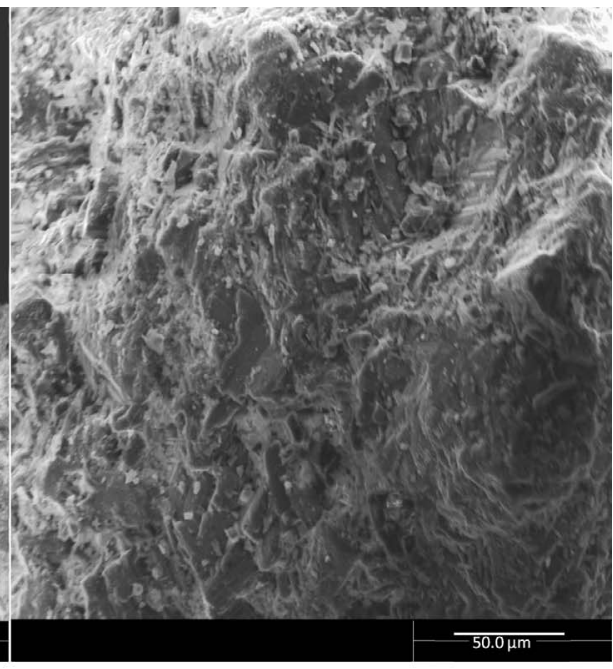

(c)

FIG. 8. Scanning electron microscopy images of filler particles (mannitol) with sizes of (a) $91 \mu \mathrm{m}$, (b) $211 \mu \mathrm{m}$, and (c) $450 \mu \mathrm{m}$.

are irregularly shaped and exhibit surface roughness. When compression is applied to form a tablet, these irregularities can lead to voids between surfaces that act as scatterers. ${ }^{41,42}$ Compared to the original particles, these voids are small enough to create wavelengthdependent scattering of the light.

Effects of Filler Particle Size and Compression Force. The scattering amplitude is plotted against the scattering slope in Fig. 9 for three different compression forces. The cluster of data for lower $\beta$ values presents tablets with FPS2 $=450 \mu \mathrm{m}$, clearly distinguishable from FPS1 and FPS3, clustered at higher $\beta$ values. This illustrates the ability of PTOFS to directly differentiate between FPS and CF.

To have a better visualization of different groups of data, a relative scale image is presented in Fig. 10a. Subtracting the fitted equation for CF1 from all fitted equations gives a clear separation of groups on the $y$ axis. Tablets with FPS2 $=450 \mu \mathrm{m}$ are indicated by filled symbols and FPS $1=91 \mu \mathrm{m}$ by open symbols; the green stars show FPS3 $=211 \mu \mathrm{m}$. Using PCA provides a similar way to explore these effects in transmission NIRS data. Figure 10b shows the PCA scatter plot for transmission NIRS data for first two principal components, explaining more than $98 \%$ variance (compared to $80 \%$ in the PTOFS data; see Fig. 5). It is interesting that the tablets made with FPS3 $=211 \mu \mathrm{m}$ and FPS1 $=91 \mu \mathrm{m}$ are located together in both figures. The reason is believed to be the smaller final size of the filler particles after tableting. The compression force affects the internal structure of the tablet depending on the properties of the particles. Thus, particle size is not the only relevant factor; particle density also affects scattering. Harder particles may not collapse as easily as softer particles under an external force. Larger particles, $450 \mu \mathrm{m}$, were made by granulation while the smaller particles were spray dried. This may explain why the tablets made with $\mathrm{FPS} 3=211 \mu \mathrm{m}$

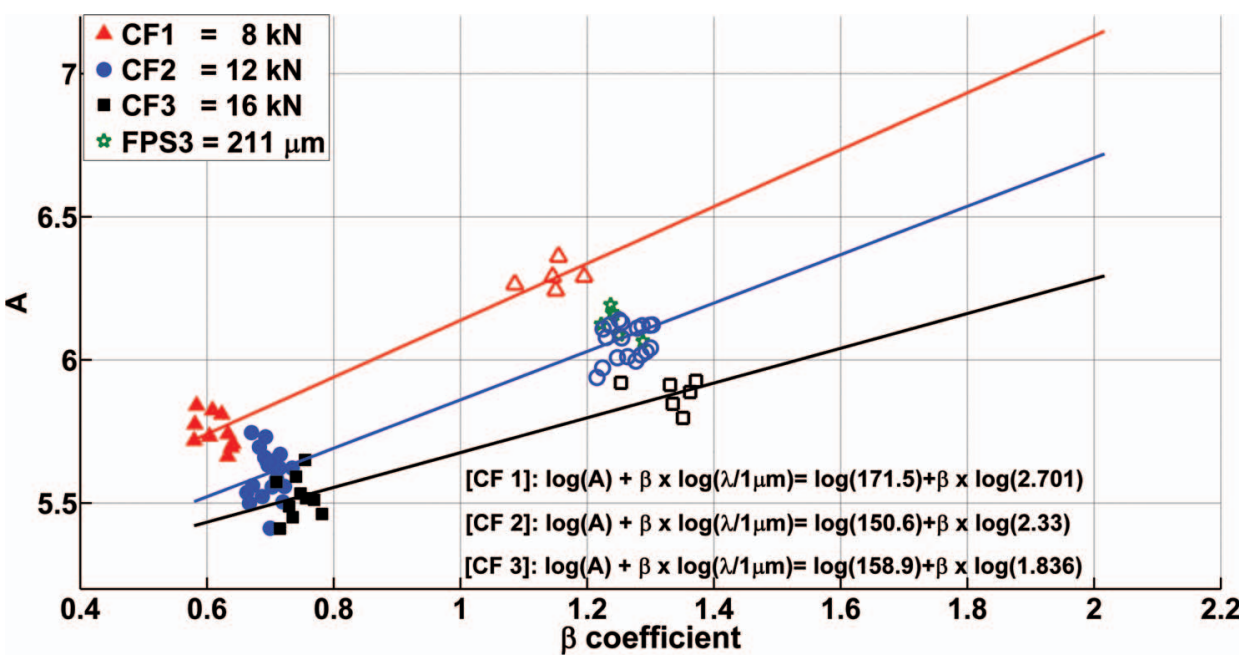

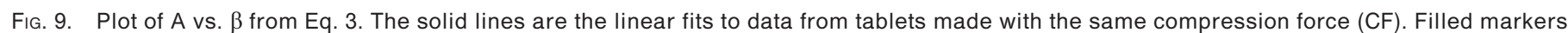

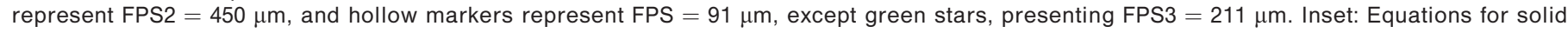
lines through each CF. 

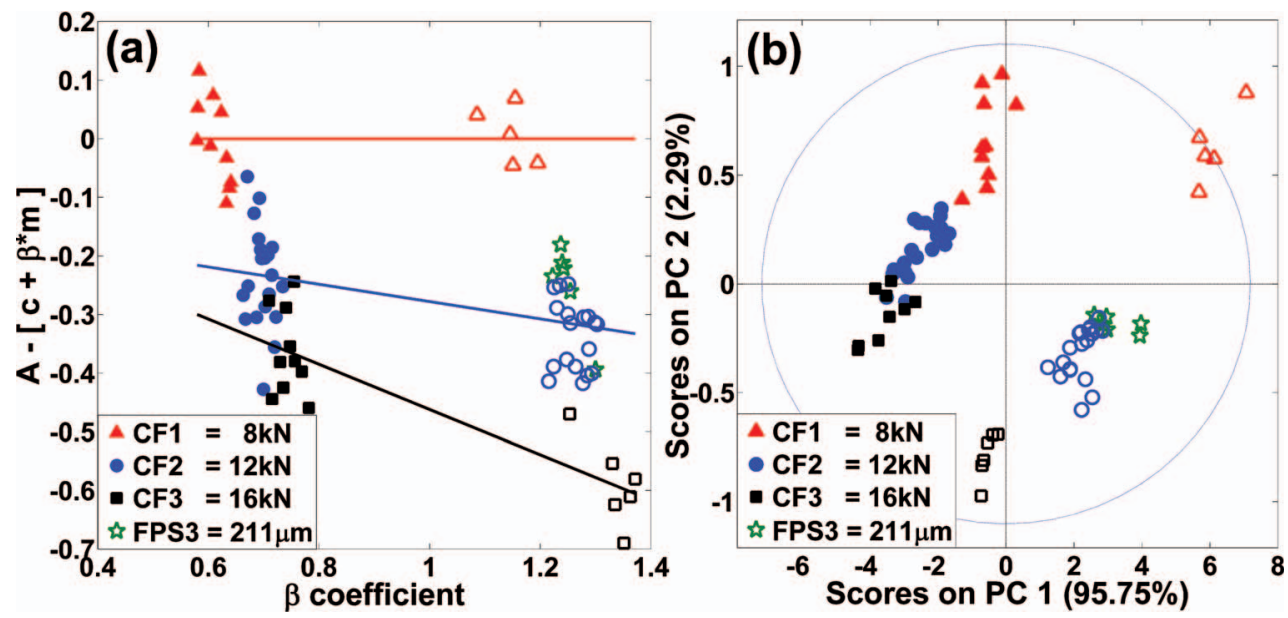

FIG. 10. (a) Rescaled plot of "A" vs. " $\beta$ " from Eq. 3 with the CF1 fit subtracted. "c" is the offset and " $m$ " is the slope of the fit. (b) PCA scatter plot of transmission NIRS data on first two principal components.

particles coincide with the particle cluster representing $\mathrm{FPS} 1=91 \mu \mathrm{m}$.

Principal component analysis as applied to the transmission NIRS data also shows a clear dependence on FPS and compression force. However, to be able to use this information in a productive manner, multivariate data analysis techniques are required for interpretation and predictions. The same information can easily be obtained from the reduced scattering coefficient of PTOFS. The effects of the production method of filler particle, particle size after compression, and compression force used during tablet production are evident in the wavelength dependence and amplitude of the PTOFS scattering spectra. Measuring the reduced scattering with PTOFS enables a direct relation to be established between particle size and the coefficient $\beta$. In this study, we observed that as the FPS increases, the effects of the compression force become less distinguishable. However, compression effects are well distinguished for tablets with lower FPSs. PTOFS can directly differentiate between filler particles made with different manufacturing processes.

\section{CONCLUSIONS}

A comprehensive study of pharmaceutical tablets has been carried out in the close NIR spectral regime 1050$1350 \mathrm{~nm}$ using PTOFS and the results compared to those obtained with traditional transmission NIR spectroscopy. Chemometric models were built to predict the ibuprofen concentration from the PTOFS absorption spectra and transmission NIRS spectra. Transmission NIRS and PTOFS were found to have comparable prediction ability when the models were based on uniformly distributed data sets. When the models were trained on tablets with larger filler particle size and challenged with test sets consisting of tablets with a smaller filler particle size, the prediction ability of transmission NIRS was significantly reduced. However, corresponding models based on PTOFS data showed better prediction ability. Models based on PTOFS absorption spectra for the prediction of drug concentrations are less sensitive to variations in filler particle size, making PTOFS a comparatively robust technique.

We showed that the amplitude and slope of reduced scattering spectra not only distinguish between manufacturing processes of filler material, but also characterize the process parameters, like compression force. PTOFS thus provides physically meaningful parameters without chemometric models. Transmission NIRS, on the other hand, provides chemometric pseudo-variables that can be difficult to interpret. The steep scattering slopes observed suggest that scattering occurs from much smaller scatterers than the original filler particles and that the observed tablet scattering spectra cannot be directly explained by the original particle size of the ingredients.

\section{ACKNOWLEDGMENTS}

This work was financed by the Centre for Imaging Food Quality project, which is funded by the Danish Council for Strategic Research (contract no. 09-067039) within the Programme Commission on Health, Food and Welfare. We are grateful to NKT Photonics for providing the light delivery system and to M. Hartman for preparing the pharmaceutical tablets, which were provided by AstraZeneca R\&D, Mölndal, Sweden.

1. J. Munson, C.F. Stanfield, B. Gujral. "A Review of Process Analytical Technology (PAT) in the U.S. Pharmaceutical Industry". Curr. Pharm. Anal. 2006. 2(4): 405-414.

2. M. Blanco, J. Coello, H. Iturriaga, S. Maspoch, C. de la Pezuela. "Near-Infrared Spectroscopy in the Pharmaceutical Industry". Analyst (Cambridge, UK). 1998. 123: 135R-150R.

3. B.F. MacDonald, K.A. Prebble. "Some Applications of Near-Infrared Reflectance Analysis in the Pharmaceutical Industry". J. Pharm. Biomed. Anal. 1993. 11(11-12): 1077-1085.

4. M. Blanco, M. Alcala. "Use of Near-Infrared Spectroscopy for OffLine Measurements in the Pharmaceutical Industry". In: K. Bakeev, editor. Process Analytical Technology. Oxford: Blackwell, 2005. Pp. 362-391.

5. Y. Roggo, P. Chalus, L. Maurer, C. Lema-Martinez, A. Edmond, N. Jent. "A Review of Near Infrared Spectroscopy and Chemometrics in Pharmaceutical Technologies". J. Pharm. Biomed. Anal. 2007. 44(3): 683-700.

6. R.C. Lyon, D.S. Lester, E.N. Lewis, E. Lee, X.Y. Lawrence, E.H. Jefferson, A.S. Hussain. "Near-Infrared Spectral Imaging for Quality Assurance of Pharmaceutical Products: Analysis of Tablets to Assess Powder Blend Homogeneity". AAPS PharmSciTech. 2002. 3(3): 1-15 
7. T. De Beer, A. Burggraeve, M. Fonteyne, L. Saerens, J.P. Remon, C. Vervaet. "Near Infrared and Raman Spectroscopy for the InProcess Monitoring of Pharmaceutical Production Processes". Int. J. Pharm. 2011. 417(1-2): 32-47.

8. A. Bogomolov. "Multivariate Process Trajectories: Capture, ResoIution and Analysis". Chemom. Intell. Lab. Syst. 2011. 108(1): 49-63.

9. E. Otsuka, H. Abe, M. Aburada, M. Otsuka. "Nondestructive Prediction of the Drug Content of an Aspirin Suppository by NearInfrared Spectroscopy". Drug Dev. Ind. Pharm. 2010. 36(7): 839-844.

10. M. Otsuka, F. Kato, Y. Matsuda. "Determination of Indomethacin Polymorphic Contents by Chemometric Near-Infrared Spectroscopy and Conventional Powder X-Ray Diffractometry". Analyst. 2001. 126(9): 1578-1582.

11. J.P. Higgins, S.M. Arrivo, G. Thurau, R.L. Green, W. Bowen, A. Lange, A.C. Templeton, D.L. Thomas, R.A. Reed. "Spectroscopic Approach for On-Line Monitoring of Particle Size During the Processing of Pharmaceutical Nanoparticles". Anal. Chem. 2003. 75(8): 1777-1785

12. M. Otsuka. "Comparative Particle Size Determination of Phenacetin Bulk Powder by Using Kubelka-Munk Theory and Principal Component Regression Analysis Based on Near-Infrared Spectroscopy". Powder Technol. 2004. 141(3): 244-250.

13. R.R. Shinde, G.V. Balgi, S.L. Nail, E.M. Sevick-Muraca. "FrequencyDomain Photon Migration Measurements for Quantitative Assessment of Powder Absorbance: A Novel Sensor of Blend Homogeneity". J. Pharm. Sci. 1999. 88(10): 959-966.

14. B.G. Osborne. "Near-Infrared Spectroscopy in Food Analysis". In R.A. Meyers, editor. Encyclopedia of Analytical Chemistry. Hoboken, NJ: John Wiley \& Sons, 2006. Online ed. doi: 10.1002/ 9780470027318.a1018

15. E. Wolfrum, C. Payne, T. Stefaniak, W. Rooney, N. Dighe, B. Bean, J Dahlberg. Multivariate Calibration Models for Sorghum Composition Using Near-Infrared Spectroscopy. Golden, CO: National Renewable Energy Laboratory, 2013.

16. Z. Sun, S. Torrance, F.K. McNeil-Watson, E.M. Sevick-Muraca. "Application of Frequency Domain Photon Migration to Particle Size Analysis and Monitoring of Pharmaceutical Powders". Anal. Chem. 2003. 75(7): 1720-1725.

17. C. Abrahamsson, A. Löwgren, B. Strömdahl, T. Svensson, S. Andersson-Engels, J. Johansson, S. Folestad. "Scatter Correction of Transmission Near-Infrared Spectra by Photon Migration Data: Quantitative Analysis of Solids". Appl. Spectrosc. 2005. 59(11): 1381-1387.

18. O.H.A. Nielsen, A.A. Subash, F.D. Nielsen, A.B. Dahl, J.L. Skytte, S. Andersson-Engels, D. Khoptyar. "Spectral Characterization of Dairy Products Using Photon Time-of-Flight Spectroscopy". J. Near Infrared Spectrosc. 2013. 21(5): 375-383.

19. A. Rinnan, F. van den Berg, S.B. Engelsen. "Review of the Most Common Pre-Processing Techniques for Near-Infrared Spectra". Trends Anal. Chem. 2009. 28(10): 1201-1222.

20. J. Mi, L. Zhang, L. Zhao, J. Li. "Particle Size Regression Correction for NIR Spectrum Based on the Relationship Between Absorbance and Particle Size". Front. Optoelectron. 2013. 6(2): 216-223.

21. T. Svensson, E. Alerstam, D. Khoptyar, J. Johansson, S. Folestad, S. Andersson-Engels. "Near-Infrared Photon Time-of-Flight Spectroscopy of Turbid Materials Up to $1400 \mathrm{~nm}$ ". Rev. Sci. Instrum. 2009. 80(6): 063105-063105.

22. C. Abrahamsson, T. Svensson, S. Svanberg, S. Andersson-Engels, J. Johansson, S. Folestad. "Time and Wavelength Resolved Spectroscopy of Turbid Media Using Light Continuum Generated in a Crystal Fiber". Opt. Express. 2004. 12(17): 4103-4112.

23. I. Bargigia, A. Tosi, A.B. Shehata, A.D. Frera, A. Farina, A. Bassi, P. Taroni, A. Dalla Mora, F. Zappa, R. Cubeddu, A. Pifferi. "TimeResolved Diffuse Optical Spectroscopy Up to $1700 \mathrm{~nm}$ by Means of a
Time-Gated IngaAs/InP Single-Photon Avalanche Diode". Appl. Spectrosc. 2012. 66(8): 944-950.

24. J. Johansson, S. Folestad, M. Josefson, A. Sparén, C. Abrahamsson, S. Andersson-Engels, S. Svanberg. "Time-Resolved NIR/VIis Spectroscopy for Analysis of Solids: Pharmaceutical Tablets". Appl. Spectrosc. 2002. 56(6): 725-731.

25. C. Abrahamsson, J. Johansson, S. Andersson-Engels, S. Svanberg, S. Folestad. "Time-Resolved NIR Spectroscopy for Quantitative Analysis of Intact Pharmaceutical Tablets". Anal. Chem. 2005. 77(4) 1055-1059.

26. D. Khoptyar, A.A. Subash, S. Johansson, M. Saleem, A. Sparén, J. Johansson, S. Andersson-Engels. "Broadband Photon Time-ofFlight Spectroscopy of Pharmaceuticals and Highly Scattering Plastics in the VIS and Close NIR Spectral Ranges". Opt. Express. 2013. 21(18): 20941-20953.

27. C. D'Andrea, E.A. Obraztsova, A. Farina, P. Taroni, G. Lanzani, A. Pifferi. "Absorption Spectroscopy of Powdered Materials Using Time-Resolved Diffuse Optical Methods”. Appl. Opt. 2012. 51(32) 7858-7863.

28. N. Souihi, M. Dumarey, H. Wikström, P. Tajarobi, M. Fransson, O. Svensson, M. Josefson, J. Trygg. "A Quality by Design Approach to Investigate the Effect of Mannitol and Dicalcium Phosphate Qualities on Roll Compaction". Int. J. Pharm. 2013. 447(1-2): 47-61.

29. A. Spare'n, O. Svensson, M. Hartman, M. Fransson, J. Johansson. "Matrix Effects in Quantitative Assessment of Pharmaceutical Tablets, Using Transmission Raman and NIR Spectroscopy". Appl. Spectrosc. Paper submitted. 2014.

30. D. Contini, F. Martelli, G. Zaccanti. "Photon Migration Through a Turbid Slab Described by a Model Based on Diffusion Approximation. I. Theory". Appl. Opt. 1997. 36(19): 4587-4599.

31. L. Eriksson, E. Johansson, N. Kettaneh-Wold, S. Wold. Introduction to Multi and Megavariate Data Analysis Using Projection Methods (PCA and PLS). Umea, Sweden: Umetrics AB, 1999

32. T. Hastie, R. Tibshirani, J. Friedman, J. Franklin. "The Elements of Statistical Learning: Data Mining, Inference and Prediction". Math. Intell. 2005. 27(2): 83-85.

33. A. Savitzky, M.J.E. Golay. "Smoothing and Differentiation of Data by Simplified Least Squares Procedures". Anal. Chem. 1964. 36(8): 1627-1639.

34. P. Geladi, D. MacDougall, H. Martens. "Linearization and ScatterCorrection for Near-Infrared Reflectance Spectra of Meat". Appl. Spectrosc. 1985. 39(3): 491-500.

35. R.J. Barnes, M.S. Dhanoa, S.J. Lister. "Standard Normal Variate Transformation and De-Trending of Near Infrared Diffuse Reflectance Spectra". Appl. Spectrosc. 1989. 43(5): 772-777.

36. E. Alerstam, S. Andersson-Engels, T. Svensson. "Improved Accuracy in Time-Resolved Diffuse Reflectance Spectroscopy". Opt. Express. 2008. 16(14): 10440-10454.

37. G.D. Batten. "Plant Analysis Using Near Infrared Reflectance Spectroscopy: The Potential and the Limitations". Aust. J. Exp. Agric. 1998. 38(7): 697-706.

38. T. Næs, T. Isaksson. "SEP or RMSEP, Which Is Best?" NIR News. 1991. 2(4): 16-0.

39. S.L. Jacques. "Fractal Nature of Light Scattering in Tissues". J. Innov. Opt. Health Sci. 2011. 4(1): 1-7

40. R. Graaff, J.G. Aarnoudse, J.R. Zijp, P.M.A. Sloot, F.F.M. de Mul, J. Greve, M.H. Koelink. "Reduced Light Scattering Properties for Mixtures of Spherical Particles: A Simple Approximation Derived from Mie Calculations". Appl. Opt. 1992. 31(10): 1370-1376.

41. W.C. Mundy, J.A. Roux, A.M. Smith. "Mie Scattering by Spheres in an Absorbing Medium". JOSA. 1974. 64(12): 1593-1597.

42. A.H. Hielscher, R.E. Alcouffe, R.L. Barbour. "Comparison of FiniteDifference Transport and Diffusion Calculations for Photon Migration in Homogeneous and Heterogeneous Tissues". Phys. Med Biol. 1998. 43(5): 1285-1302. 